\title{
APRECIACIÓN ESTUDIANTIL DE LA INVESTIGACIÓN MÉDICA EN PREGRADO. ESTUDIO MULTICÉNTRICO EN 19 UNIVERSIDADES
}

\author{
STUDENT APPRECIATION OF MEDICAL RESEARCH IN UNDERGRADUATE. \\ MULTICENTER STUDY IN 19 UNIVERSITIES \\ Mayron D. Nakandakari ${ }^{1, a}$
}

\begin{abstract}
RESUMEN
Objetivo: Conocer la apreciación estudiantil sobre la labor de las universidades peruanas en la investigación científico médica de pregrado en estudiantes de facultades de medicina del Perú, 2016-2017. Métodos: El estudio fue de tipo multicéntrico, observacional, descriptivo, transversal. Se contó con la participación de 400 estudiantes de medicina pertenecientes a 19 facultades de medicina reconocidas oficialmente por la Asociación Peruana de Facultades de Medicina (ASPEFAM). El muestreo aplicado fue no probabilístico. Se utilizó una encuesta validada por un estudio anterior, autoaplicativa, anónima, voluntaria, y que fue sometida a una prueba piloto. La recolección fue virtual y el análisis realizado fue de tipo descriptivo. Resultados: De la población encuestada, solo el 7,8\% refirió que su facultad de medicina sí apoyaba a la investigación. Alrededor del $56 \%$ refirió no sentirse suficientemente capacitado por su universidad para lograr la publicación científica en revistas biomédicas. Y en su gran mayoría hicieron apreciar que en sus universidades no se fomentan los semilleros en investigación, no cuentan con pasantías científicas y no existe apoyo para que los estudiantes ejecuten o realicen sus tesis. Conclusión: La apreciación de los estudiantes entorno al trabajo que vienen haciendo las universidades $y / o$ facultades de medicina en la investigación científico médica de pregrado, fue catalogada como "deficiente" por la mayoría de los encuestados.
\end{abstract}

Palabras clave: Investigación médica; Educación de pregrado en medicina; Estudiantes de medicina; Perú. (fuente: DeCS BIREME)

\begin{abstract}
Objective: To know the appreciation of the student on the work of the Peruvian scientific research universities medical undergraduate students of faculties of medicine of the Peru, 2016-2017. Methods: It is a multicentric, observational, descriptive and transversal study. It featured the participation of 400 medical students belonging to 19 faculties of medicine officially recognized by the Peruvian Association of faculties of Medicine (ASPEFAM). Applied sampling was not probabilistic. A validated by a study earlier, auto-applicative, anonymous, voluntary survey was used, and which underwent a pilot test. The collection was virtual and the analysis was descriptive. Results: Only 7,8\% concerned that its faculty of medicine Yes supported research. Around $56 \%$ concerned do not feel sufficiently trained by their University to achieve the scientific publication in biomedical journals. And most they did appreciate that their universities do not encourage the seed research, do not have scientific internships and there is support for students to execute or carry out their thesis. Conclusions: The perceptions of the students about the work of universities in undergraduate scientific medical research, it was classified as "deficient" by the majority of the surveyed.
\end{abstract}

Key words: Medical research; Undergraduate medical education; Medical students; Peru. (source: MeSH NLM)

${ }^{1}$ Universidad Privada San Juan Bautista, Lima-Perú.

a Médico Cirujano.

Citar como: Mayron D. Nakandakari. Apreciación estudiantil de la investigación médica en pregrado. Estudio multicéntrico en 19 universidades. Rev. Fac. Med. Hum. Octubre 2019;19(4):74-83. DOI 10.25176/RFMH.v19i4.2337

Artículo publicado por la Revista de la Facultad de Medicina Humana de la Universidad Ricardo Palma. Es un artículo de acceso abierto, distribuído bajo los términos de la Licencia Creative Commons: Creative Commons Attribution 4.0 International, CC BY 4.0 (https://creativecommons.org/licenses/by/4.0/), que permite el uso no comercial, distribución y reproducción en cualquier medio, siempre que la obra original sea debidamente citada. Para uso comercial, por favor póngase en contacto con revista.medicina@urp.pe 


\section{INTRODUCCIÓN}

La investigación científica puede ser definida como toda actividad humana orientada a la obtención de nuevos conocimientos en pro de la solución de brechas o problemas que existen en la realidad. Esta actividad es inherente a la medicina humana, por ello todo estudiante de la profesión médica tiene que conocer sobre investigación, y para ello necesita ser formado'. Es erróneo el pensar que los estudiantes de pregrado no pueden hacer investigación ni publicar sus artículos en revistas científico médicas nacionales y extranjeras. Evidencia de esto son las innumerables publicaciones que vienen logrando estudiantes peruanos y de todas partes del mundo ${ }^{2-4}$.

Según el ranking de Scimago en su informe SIR 2015, el Perú tiene 72 instituciones universitarias, las cuales juntas tuvieron una producción científica de 4311 artículos. Incluso las universidades mejor situadas en el ranking nacional de publicaciones, no tienen punto de comparación con el número de publicaciones a nivel mundial; estando en peor situación las universidades ubicadas en puestos más bajos del ranking, que inclusive llegan a tener una producción científica nula ${ }^{5}$. Así pues, acorde a la ley universitaria $N^{\circ} 30220$ peruana promulgada en el año 2014, "la universidad peruana tiene como una de sus funciones esenciales y obligatorias la investigación científica realizada tanto por docentes, egresados y estudiantes de pregrado"6, especialmente en el campo de la medicina humana, por tanto, se planteó como objetivo general conocer la apreciación estudiantil sobre la labor de las universidades peruanas en la investigación científico médica de pregrado en estudiantes de Facultades de Medicina del Perú, 2016-2017.

\section{MÉTODOS}

\section{Tipo y diseño del estudio}

Estudio de tipo multicéntrico, observacional, descriptivo y transversal, ejecutándose en noviembre del 2016 hasta enero del 2017. Asimismo, se realizó una convocatoria para la participación de estudiantes de medicina de las 25 universidades reconocidas por la Asociación Peruana de Facultades de Medicina (ASPEFAM).

\section{Población y muestra}

La población estudiada fueron todos los estudiantes de las Facultades de Medicina Peruanas reconocidas oficialmente por ASPEFAM, 2016. El tipo de muestreo fue no probabilístico por conveniencia.
Se incluyeron a todos aquellos estudiantes de medicina que hayan llevado por lo menos en su currícula universitaria, un curso que esté relacionado a investigación, queestén matriculados en algún semestre universitario y que perteneciesen a una facultad $y / o$ escuela de Medicina reconocida oficialmente por ASPEFAM.

Se excluyeron a todos aquellos estudiantes que fuesen parte de alguna Sociedad Científica Universitaria y a todos los que no deseasen ser partícipes del estudio.

\section{Recolección de datos}

Se utilizó una encuesta que ya fue validada por un estudio anterior ${ }^{18}$, autoaplicativa, anónima, voluntaria, que fue sometida a una prueba piloto conformada por 64 participantes y a la cual se le calculó un coeficiente de alfa de Cronbach de 0,91 indicativo de una alta confiabilidad del instrumento .

La encuesta constó de seis secciones: 1) Datos personales y apreciación sobre la labor universitaria en investigación (07 ítems), 2) Actividades y cursos universitarios relacionados a la investigación (08 ítems), 3) Estrategias, políticas y técnicas de promoción en investigación (20 ítems), 4) Premiación y reconocimiento a los investigadores de pregrado (04 ítems).

Para encuestar, se buscó establecer contacto con al menos un representante de las distintas Sociedades Científicas de las universidades peruanas, el cual contactase a los estudiantes de medicina de sus facultades y/o escuelas de medicina humana para que fuesen partícipes del estudio.

La recolección de datos se realizó desde noviembre del 2016 hasta enero del 2017, de manera virtual, a través del envío de un correo electrónico a cada uno de los participantes, donde se anexó la encuesta autoaplicativa con el consentimiento informado.

\section{Variables}

La variable fue la "labor universitaria en investigación científico médica de pregrado", la cual fue estudiada en base a: a) Actividades y cursos universitarios sobre investigación, b) Investigación por los docentes y los directivos universitarios, c) Premiación y reconocimiento a los investigadores de pregrado.

\section{Análisis estadístico}

El análisis de las variables fue de nivel descriptivo. Los datos fueron ingresados a una base diseñada para el estudio en los paquetes estadísticos Microsoft Excel $2013^{\circledast}$. 


\section{Consideraciones éticas}

El presente trabajo fue aprobado para su realización por el Comité de Ética en Investigación de la Universidad Privada San Juan Bautista (UPSJB).

Así mismo, para garantizar la ética en este estudio, se brindó el consentimiento informado. Además, el manejo de sus datos personales fue a través de códigos numerales, y todo dato recolectado estuvo bajo custodia única del investigador, quien veló por la mayor confidencialidad posible en todos los procesos de la investigación.

\section{RESULTADOS}

Se obtuvo respuesta de estudiantes de 19 universidades reconocidas por ASPEFAM de las 25 existentes: nueve nacionales y diez privadas, con un promedio de 21 estudiantes por cada facultad de medicina, llegando a una población encuestada de 400 estudiantes. El $57,8 \%$ pertenecían a universidades particulares, y solo el 23,6\% estudiaban en universidades de Lima. Respecto a la apreciación de los estudiantes sobre la labor que vienen haciendo sus universidades entorno a la investigación de pregrado, lo valoraron en un $78,8 \%$ como una labor aún "deficiente" (Tabla 1).

Tanto la mayoría de estudiantes de universidades nacionales como privadas estuvieron de acuerdo que sus casas de estudio realizaban actividades científicas como conferencias de investigación, jornadas o congresos científicos, así como también programaban dentro de la currícula cursos relacionados a investigación, no obstante, en alrededor del 79,3\% de los encuestados hay ausencia de cursos extracurriculares sobre investigación en sus universidades.

Así mismo, el 56,3\% de los estudiantes no se sienten lo suficientemente capacitados para lograr la publicación científica únicamente con los cursos sobre investigación que le imparte su facultad o escuela de medicina.
Por otro lado, un alto porcentaje refirió que la mayoría de proyectos que son realizados en la vida universitaria, solo son hechos como parte de la nota final de los cursos, y mas no para participar en distintos eventos científicos ni para su publicación (Tabla 2).

En la Tabla 3, se muestran las apreciaciones que tienen los estudiantes de medicina sobre los cursos curriculares y extracurriculares que brindan sus universidades, tanto a nivel privado como público, calificando en su mayoria a estos cursos curriculares de una calidad regular, no obstante, también existió un pequeño grupo que llegó a calificar dichos cursos como deficientes o nulos. El curso curricular que más frecuentemente llevaron fue el de metodología de investigación. Respecto a los cursos extracurriculares, solo el $20,8 \%$ del total llevó uno de estos cursos y mayoritariamente lo calificaron con una calidad de regular.

Por otro lado, también se consultó si los estudiantes sabían si sus docentes realizaban investigación, a esto la mayoría refirió que solo "algunos" de los docentes que les habían dictado los cursos en su facultad, hacían investigación científica, asi como haber publicado investigaciones originales en revistas científicas médicas. Así mismo, el 76,5 \% de los encuestados alguna vez intentaron realizar ya sea un proyecto o un trabajo de investigación con apoyo o asesoramiento de sus docentes universitarios, recibiendo en su gran mayoría apoyo de ellos sin costo alguno (Tabla 4).

Sobre la premiación, reconocimiento a los investigadores, políticas y estrategias universitarias para la investigación científica, alrededor del $48 \%$ de los encuestados refirieron que sus universidades no daban ningún premio y/o reconocimiento a aquellos estudiantes y docentes que lograban la publicación de artículos de investigación en Revistas Científicas Médicas, así mismo el $54,7 \%$ mencionó que no se hacían difusión de dichas publicaciones. 
Tabla 1. Características generales de los estudiantes de medicina participantes y sus apreciaciones sobre la labor de sus universidades entorno a la investigación científica de pregrado $(n=400)$.

\begin{tabular}{ccccccc}
\hline Tipos de universidades/ & \multicolumn{2}{c}{ Nacional } & \multicolumn{2}{c}{ Privada } & \multicolumn{2}{c}{ Total } \\
Características de los estudiantes & $\mathbf{N}^{\circ}$ & $(\%)$ & $\mathbf{N}^{\circ}$ & $(\%)$ & $\mathbf{N}^{\circ}$ & $(\%)$
\end{tabular}

Características generales y apreciación

1. Ubicación de su universidad:

$\begin{array}{lcccccc}\text { Lima } & 19 & 4,8 & 75 & 18,8 & 94 & 23,6 \\ \text { Otros } & 150 & 37,6 & 156 & 39 & 306 & 76,6\end{array}$

2. Edad:

\begin{tabular}{|c|c|c|c|c|c|c|}
\hline Media & 21,4 & - & 21,7 & - & 21,5 & - \\
\hline Mediana & 21 & - & 21 & - & 21 & - \\
\hline Moda & 19 & - & 21 & - & 21 & - \\
\hline [Mínimo-máximo] & [18-30] & - & [17-31] & - & [17-31] & - \\
\hline \multicolumn{7}{|l|}{ 3. Género: } \\
\hline Masculino & 107 & 26,8 & 94 & 23,6 & 201 & 50,4 \\
\hline Femenino & 62 & 15,6 & 137 & 34,3 & 199 & 49,9 \\
\hline \multicolumn{7}{|c|}{ 4. Año de estudios universitarios: } \\
\hline Primero & 19 & 4,7 & 19 & 4,7 & 38 & 9,4 \\
\hline Segundo & 19 & 4,7 & 25 & 6,3 & 44 & 11 \\
\hline Tercero & 44 & 10,9 & 25 & 6,3 & 69 & 17,2 \\
\hline Cuarto & 24 & 6 & 56 & 14,1 & 80 & 20,1 \\
\hline Quinto & 44 & 10,9 & 75 & 18,8 & 119 & 29,7 \\
\hline Sexto & 19 & 4,7 & 31 & 7,8 & 50 & 12,5 \\
\hline \multicolumn{7}{|c|}{ 5. Valoración de universidad en investigación } \\
\hline a. Muy buena & 0 & 0 & 0 & 0 & 0 & 0 \\
\hline b. Buena & 4 & 1 & 2 & 0,5 & 6 & 1,5 \\
\hline c. Regular & 45 & 11,3 & 31 & 7,8 & 76 & 19,1 \\
\hline d. Deficiente & 133 & 33,3 & 182 & 45,5 & 315 & 78,8 \\
\hline e. Muy deficiente & 1 & 0,3 & 2 & 0,5 & 3 & 0,8 \\
\hline
\end{tabular}

Fuente: Elaboración propia del autor según la encuesta “Apreciación estudiantil sobre la labor de las universidades peruanas en la investigación científico médica de pregrado." 
Tabla 2. Actividades científicas sobre investigación que realizan las facultades de medicina $(n=400)$.

\begin{tabular}{cccccccc}
\hline $\begin{array}{c}\text { Tipos de universidades/actividades } \\
\text { cientificas }\end{array}$ & \multicolumn{2}{c}{ Nacional } & \multicolumn{2}{c}{ Privada } & \multicolumn{2}{c}{ Total } \\
& $\mathbf{N}^{\circ}$ & $(\%)$ & $\mathbf{N}^{\circ}$ & $(\%)$ & $\mathbf{N}^{\circ}$ & $(\%)$
\end{tabular}

Sobre las actividades científicas organizadas por las facultades

1. ¿Se hacen actividades científicas como conferencias sobre investigación, jornadas científicas o congresos científicos?
a. Sí
93
23,2
187
46,8
280
70
b. No
75
18,8
32
8
107
26,8
c. Desconozco
0
0
13
3,2
13
3,2

2. Dentro de la currícula de su facultad o escuela de medicina, ¿usted lleva algún curso relacionado a la Investigación?
a. Sí
170
42,4
230
57,6
400
100

3. ¿Su facultad o escuela de medicina realiza cursos extracurriculares de investigación?
a. Sí
23
5,8
60
15
83
20,8
b. No
168
42
149
37,3
317
79,3

4. Con las clases de su facultad ¿cree usted estar capacitado para lograr la publicación científica?
a. Totalmente de acuerdo
0
0
0
$0 \quad 0$
0
b. De acuerdo
6
56
14
62
15,5
c. Ni de acuerdo ni en desacuerdo
38
$9,5 \quad 75$
18,8
113
28,3
d. Desacuerdo
88
22
67
16,8
155
38,8
e. Totalmente en desacuerdo
38
9,5
32
8
70
17,5

5. ¿Se hacen actividades extracurriculares en el que se ejecuten proyectos de investigación?
a. Sí
43
10,8
62
15,5
105
26,3
b. No
$112 \quad 28,0$
127
31,8
239
59,8
c. Desconozco
13
3,3
43
10,8
56
14,1

6. ¿Qué ocurre con los proyectos de investigación que son ejecutados en las actividades extracurriculares?

a. Se convierten en trabajos que presentamos para nota del curso

25

6,3

42

10,5

67

16,8

b. Se convierten en trabajos con los que participamos en eventos científicos como

congresos y jornadas científicas

c. Se convierten en trabajos investigación que los enviamos a publicar a revistas

0

0,0

0

0,0

0

0 científicas

d. Desconozco

6

1,5

0

0,0

6

1,5

Fuente: Elaboración propia del autor según la encuesta “Apreciación estudiantil sobre la labor de las universidades peruanas en la investigación científico médica de pregrado." 
Tabla 3. Apreciación sobre los cursos universitarios relacionados a investigación que realizan las facultades de medicina $(n=400)$.

\begin{tabular}{|c|c|c|c|c|c|c|c|c|c|c|}
\hline \multirow{2}{*}{ Apreciación/ cursos } & \multicolumn{2}{|c|}{ Muy Bueno } & \multicolumn{2}{|c|}{ Bueno } & \multicolumn{2}{|c|}{ Regular } & \multicolumn{2}{|c|}{ Deficiente } & \multicolumn{2}{|c|}{$\begin{array}{c}\text { Muy } \\
\text { deficiente }\end{array}$} \\
\hline & $\begin{array}{l}\text { UN* } \\
(\%)\end{array}$ & $\begin{array}{l}\text { UP** } \\
\text { (\%) }\end{array}$ & $\begin{array}{l}\text { UN } \\
(\%)\end{array}$ & $\begin{array}{l}\text { UP } \\
\text { (\%) }\end{array}$ & $\begin{array}{l}\text { UN } \\
(\%)\end{array}$ & $\begin{array}{l}\text { UP } \\
(\%)\end{array}$ & $\begin{array}{l}\text { UN } \\
(\%)\end{array}$ & $\begin{array}{l}\text { UP } \\
(\%)\end{array}$ & $\begin{array}{l}\text { UN } \\
(\%)\end{array}$ & $\begin{array}{l}\text { UP } \\
(\%)\end{array}$ \\
\hline
\end{tabular}

Cursos de la currícula

$\begin{array}{lcccccccccc}\begin{array}{l}\text { a. Metodología de la } \\ \text { investigación }\end{array} & 0 & 38 & 44 & 50 & 69 & 93 & 50 & 25 & 0 & 6 \\ & (0,0) & (9,5) & (11,0) & (12,5) & (17,3) & (23,3) & (12,5) & (6,3) & (0,0) & (1,5) \\ \text { b. Epidemiología } & 0 & 19 & 25 & 44 & 12 & 75 & 25 & 19 & 6 & 0 \\ & (0,0) & (4,8) & (6,3) & (11,0) & (3,0) & (18,8) & (6,3) & (4,8) & (1,5) & (0,0) \\ & 12 & 31 & 6 & 38 & 38 & 82 & 56 & 50 & 6 & 0 \\ \text { c. Estadística } & (3,0) & (7,8) & (1,5) & (9,5) & (9,5) & (20,5) & (14,0) & (12,5) & (1,5) & (0,0) \\ & 0 & 12 & 19 & 50 & 25 & 31 & 12 & 31 & 0 & 12 \\ \text { d. Ética en investigación } & (0,0) & (3,0) & (4,8) & (12,5) & (6,3) & (7,8) & (3,0) & (7,8) & (0,0) & (3,0) \\ & 0 & 0 & 12 & 19 & 19 & 31 & 19 & 12 & 6 & 6 \\ \text { e. Tesis } & (0,0) & (0,0) & (3,0) & (4,8) & (4,8) & (7,8) & (4,8) & (3,0) & (1,5) & (1,5)\end{array}$

Curso extracurricular

$\begin{array}{lcccccccccc} & 0 & 0 & 0 & 10 & 0 & 30 & 0 & 0 & 10 & 9 \\ \text { a. Redacción científica } & (0,0) & (0,0) & (0,0) & (2,5) & (0,0) & (7,5) & (0,0) & & (2,5) & (2,3) \\ & 0 & 9 & 0 & 0 & 0 & 9 & 0 & 15 & 9 & 9 \\ \text { b. Publicación científica } & (0,0) & (2,3) & (0,0) & (0,0) & (0,0) & (2,3) & (0,0) & (3,8) & (2,3) & (2,3)\end{array}$

$\begin{array}{lllllllllll}\text { c. Proyecto de investigación } & 0 & 0 & 0 & 10 & 16 & 29 & 0 & 9 & 0 & 10\end{array}$

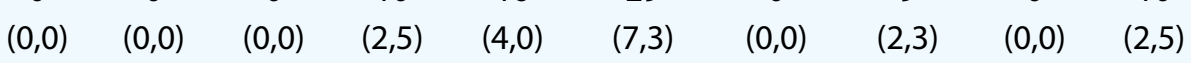

d. Metodología de la

investigación

$\begin{array}{llllllllll}0 & 9 & 0 & 9 & 0 & 29 & 9 & 9 & 0 & 9\end{array}$

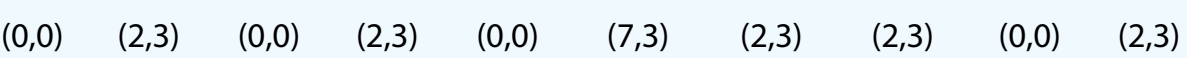

e. Manejo de SPSS

$\begin{array}{llllllllll}0 & 10 & 0 & 0 & 0 & 30 & 0 & 9 & 0 & 10\end{array}$

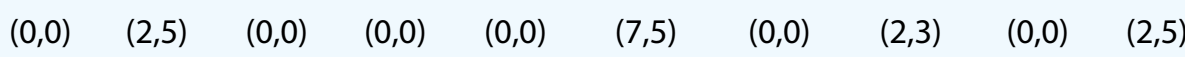

f. Manejo de STATA

$\begin{array}{llllllllll}0 & 0 & 0 & 9 & 0 & 9 & 0 & 16 & 0 & 10\end{array}$ $\begin{array}{llllllll}(0,0) & (0,0) \quad(0,0) \quad(2,3) \quad(0,0) \quad(2,3) \quad(0,0) \quad(4,0) \quad(0,0) \quad(2,5)\end{array}$

g. Ética en investigación

$\begin{array}{cccccccccc}0 & 9 & 9 & 15 & 0 & 23 & 0 & 9 & 0 & 0 \\ (0,0) & (2,3) & (2,3) & (3,8) & (0,0) & (5,8) & (0,0) & (2,3) & (0,0) & (0,0)\end{array}$

h. Lectura crítica de artículos científicos

$\begin{array}{cccccccccc}0 & 0 & 0 & 0 & 0 & 17 & 0 & 11 & 0 & 11 \\ (0,0) & (0,0) & (0,0) & (0,0) & (0,0) & (4,3) & (0,0) & (2,8) & (0,0) & (2,8)\end{array}$

*UN: Universidad Nacional, **UP: Universidad Privada

Fuente: Elaboración propia del autor según la encuesta "Apreciación estudiantil sobre la labor de las universidades peruanas en la investigación científico médica de pregrado." 
Tabla 4. Apreciación de los estudiantes sobre la labor investigadora de los docentes y directivos universitarios $(n=400)$.

\section{Tipos de universidades/ítems de la encuesta}

\section{Nacional Privada}

1. ¿Los docentes que le han dictado los cursos en su facultad o escuela de medicina hacen investigación científica?

a. Todos los docentes hacen investigación

b. La gran mayoría

c. Algunos

d. Casi ninguno

e. Ninguno

f. Desconozco

$\begin{array}{cccccc}12 & 3,1 & 62 & 15,6 & 74 & 18,7 \\ 19 & 4,7 & 56 & 14,1 & 75 & 18,8 \\ 81 & 20,3 & 88 & 21,9 & 169 & 42,2 \\ 38 & 9,4 & 12 & 3,1 & 50 & 12,5 \\ 1 & 0,3 & 6 & 1,6 & 7 & 1,9 \\ 19 & 4,7 & 6 & 1,6 & 25 & 6,3\end{array}$

2. ¿Los docentes que le han dictado los cursos en su facultad o escuela de medicina han publicado artículos de investigaciones originales en revistas científicas médicas?

$\begin{array}{lcccccc}\text { a. Todos los docentes hacen investigación } & 12 & 3,1 & 62 & 15,6 & 74 & 18,7 \\ \text { b. La gran mayoría } & 25 & 6,3 & 50 & 12,5 & 75 & 18,8 \\ \text { c. Algunos } & 69 & 17,2 & 62 & 15,6 & 131 & 32,8 \\ \text { d. Casi ninguno } & 38 & 9,4 & 19 & 4,7 & 57 & 14,1 \\ \text { e. Ninguno } & 13 & 3,3 & 6 & 1,6 & 19 & 4,9 \\ \text { f. Desconozco } & 13 & 3,3 & 31 & 7,8 & 44 & 11,1\end{array}$

3. ¿Cuántos de los docentes que a usted le enseñan promueven la investigación entre sus estudiantes en el curso que dictan?

a. La totalidad de los docentes

b. La gran mayoría

$\begin{array}{lcccccc}\text { a. La totalidad de los docentes } & 12 & 3,1 & 0 & 0 & 12 & 3,1 \\ \text { b. La gran mayoría } & 25 & 6,3 & 19 & 4,7 & 44 & 11 \\ \text { c. Algunos } & 94 & 23,4 & 156 & 39,1 & 250 & 62,5 \\ \text { d. Casi ninguno } & 12 & 3,1 & 12 & 3,1 & 24 & 6,2 \\ \text { e. Ninguno } & 13 & 3,3 & 38 & 9,4 & 51 & 12,7 \\ \text { f. Desconozco } & 13 & 3,3 & 6 & 1,6 & 19 & 4,9\end{array}$

4. ¿Las autoridades de su Universidad, sean su vicerrector (a) de investigación, director (a) de facultad o escuela, o su decano (a), tienen artículos de investigación publicados en revistas científicas médicas?
a. Sí
$\begin{array}{llllll}38 & 9,4 & 69 & 17,2 & 107 & 26,6\end{array}$
b. No
$\begin{array}{llllll}44 & 10,9 & 30 & 7,5 & 74 & 18,4\end{array}$
c. Desconozco
$88 \quad 21,9 \quad 131$
$32,8 \quad 219 \quad 54,7$

5. ¿Usted alguna vez ha intentado realizar ya sea un proyecto o un trabajo de investigación con apoyo o asesoramiento de los docentes de su facultad o escuela de medicina?
a. Sí
14435

$35,9 \quad 162$

6240,6

30676,5
b. No
256,3
$69 \quad 17,2 \quad 94$
23,5

6. ¿Sus docentes estuvieron dispuestos a participar o asesorar su proyecto o trabajo de investigación?
a. Sí
$\begin{array}{lll}126 & 31,5 & 133\end{array}$
$33,3 \quad 259$
64,8
b. No
8

$2 \quad 39$
9,8
47
11,8
7. ¿Sus docentes le cobraron monetariamente por el asesoramiento?
a. No
126
31,5
133
$33,3 \quad 259$
64,8

8. ¿Sus docentes tienen horarios fuera de las clases asignados por la facultad o escuela de medicina para asesorar e investigar con los estudiantes?

\begin{tabular}{lcccccc} 
a. Sí & 25 & 6,3 & 38 & 9,4 & 63 & 15,7 \\
b. No & 112 & 28,1 & 94 & 23,4 & 206 & 51,5 \\
c. Desconozco & 31 & 7,8 & 100 & 25 & 131 & 32,8 \\
\hline
\end{tabular}

Fuente: Elaboración propia del autor según la encuesta “Apreciación estudiantil sobre la labor de las universidades peruanas en la investigación científico médica de pregrado." 


\section{DISCUSIÓN}

Entre los principales problemas detectados a través de la apreciación de los estudiantes están la deficiente capacitación universitaria sobre investigación, el poco apoyo de la universidad a la investigación de pregrado y la no motivación a los estudiantes para que hagan ciencia. Todo ello sustenta que alrededor del $78,8 \%$ de los estudiantes, a través de las encuestas, hayan valorado como "deficiente" la labor de las universidades en la investigación de pregrado.

Referente a la deficiente capacitación, se puede mencionar que esto en su gran mayoría se identifica porque los estudiantes no se sienten suficientemente capacitados por su universidad para lograr la publicación científica, ni para realizar y sustentar su tesis ${ }^{17}$. Esto también se menciona en el estudio de Molina et al. ${ }^{18}$, donde hubo un gran grupo que consideró deficiente la currícula relacionada a investigación que las universidades brindaban, y la única salida que encontraban era buscar cursos extracurriculares, no dictados por las universidades, pero sí frecuentemente impartidos por otras entidades, los cuales según los estudiantes eran muy completos y de mayor calidad ${ }^{19-21}$.

Entorno al poco apoyo a la investigación de pregrado, es claro que las universidades no están formando recurso humano en investigación ${ }^{22,23}$. Ni brindándoles oportunidades como los fondos económicos concursables, los cuales pueden ayudar a combatir una de las más claras dificultades para hacer investigación estudiantil en el Perú, el cual es más comúnmente destinado solo a docentes universitarios según Arroyo et al. ${ }^{24-27}$.

Los proyectos, trabajos de investigación, y las tesis únicamente están formando parte de las notas de los cursos y muchas veces son un requisito más para la titulación, pero no se está incentivando a que los estudiantes publiquen este tipo de contribuciones científicas. Así mismo, no se está brindando reconocimientos ni premiando a aquellos estudiantes que obtienen logros en el ámbito científico, hecho que, si se diera, incentivaría a más estudiantes a investigar ${ }^{28,29}$.

Por otro lado, es claro que las universidades no cuentan con ambientes que motiven a la investigación, ya que los mismos laboratorios tal y como se identifica en nuestro estudio, solo están siendo utilizados para la enseñanza académica y mas no para la ejecución de estudios científicos de mayor complejidad ${ }^{30}$.

Un punto aparte es la aún no existencia de semilleros de investigación en las universidades, dado que aún existe la creencia de esperar a que los alumnos lleguen recién a los ciclos donde llevan algún curso de investigación para hacer ciencia, por esto no se les está motivando a hacer investigación desde el primer ciclo de la carrera, lo cual evidentemente sería mucho más provechoso e incrementaría las investigaciones en ciencias básicas ${ }^{31,32}$.

Dentro de las limitaciones de la investigación se encuentran: la poca accesibilidad de trabajo tanto con las facultades de medicina como con sus estudiantes, dada sus localizaciones en diferentes latitudes del Perú. Además de ello, inicialmente se tenía propuesto realizar un muestreo probabilístico estratificado por año de estudio, no obstante, hubo un alto grado de dificultad al momento de mantener contacto con cada facultad de medicina para los permisos de obtener información acerca de sus estudiantes con el fin de conocer el número total de estudiantes por cicloy/o año de carrera, así mismo, hubieron varias complicaciones durante el proceso de encuestar para poder contactar al número de estudiantes requerido por cada año de estudio y por cada facultad de medicina del Perú; por lo cual, se planteó trabajar con un muestreo no probabilístico por conveniencia, el cual quizá no fue el ideal, pero permitió abarcar estudiantes de distintos años de carrera por cada facultad, y obtener distintos puntos de apreciación sobre la labor universitaria en investigación.

Por otro lado, entre las fortalezas que presenta esta investigación se resalta ser un estudio de multicéntrico que abarcó las apreciaciones de estudiantes de 19 facultades de medicina, a diferencia de otras investigaciones que sólo recogieron información local de sus realidades universitarias.

Además, es uno de los pocos estudios a nivel nacional que ha indagado la realidad de la investigación científica en pregrado posterior a la promulgación de la ley universitaria № 30220 en el año 2014. De por sí, previa a la promulgación de la ley, no era muy bien apreciada la labor universitaria en investigación, he ahí la necesidad que se tuvo para conocer si algo había cambiado a corto y mediano plazo con la aplicación de la misma.

\section{CONCLUSIÓN}

Más del $50 \%$ de los encuestados valoran como deficiente la investigación en sus universidades durante, dado que se está demostrando a cada estudiante una mala imagen y una falta de interés por el desarrollo de ciencia. Por ello se sugiere, empezar a establecer políticas y estrategias claras en investigación, específicas para cada realidad universitaria, y que sean aplicadas desde ya. Así mismo se recomienda mejorar la difusión de los logros científicos que cada universidad obtenga, y reforzar los cursos sobre investigación desde el inicio de la carrera universitaria y promover paralelo a ello la publicación en revistas científicas. 
Contribución deautoría:MNparticipóenlaconcepción y diseño del artículo, obtención de resultados, análisis e interpretación de datos, redacción del artículo, aprobación de su versión final, aporte de pacientes o material de estudio, y asesoría administrativa.

Financiamiento recibido: Autofinanciado.
Conflicto de interés: El autor del presente trabajo de investigación declara no tener conflicto de interés.

Recibido: 02 de agosto 2019

Aprobado: 30 de agosto 2019

Correspondencia: Mayron D. Nakandakari Gómez.

Dirección: Mz. A5 Lot. 10 AAHH. Cerro Candela. San Martín de Porres. Lima, Perú.

Teléfono: (+51) 1973-868114

Correo: mayron.nakandakari@outlook.com

\section{REFERENCIAS BIBLIOGRÁFICAS}

1. Huamaní C, Chávez-Solis P, Mayta-Tristán P. Aporte estudiantil en la publicación de artículos científicos en revistas médicas indizadas en Scielo-Perú, 1997 - 2005. An Fac Med. 2008; 69 (1):42-5. Disponible en: https://doi.org/10.15381/anales.v69i1.1182

2. Alarcón-Villaverde J, Romaní F, Gutiérrez C. Publicaciones científicas estudiantiles producidas en el curso de Epidemiología de la Facultad de Medicina de la Universidad Nacional Mayor de San Marco durante el periodo 2003-2009. An Fac Med. 2010; 71: 111-6. Disponible en: https:// doi.org/10.15381/anales.v71i2.82

3. Arriola-Quiroz I, Curioso WH, Cruz-Encarnación M, Gayoso O. Characteristics and publication patterns of these from a Peruvian medical school. Health Info Libr J. 2010; 27: 148-54. Disponible en: https://doi. org/10.1111/j.1471-1842.2010.00878.x

4. Galan E, Manrique N, Villavicencio E, Yllatopa E, Peralta M, De La Cruz W. Producción científica de los investigadores del pregrado de medicina humana del Peru, 1993-2003. CIMEL. 2005, 10: 41-48. Disponible en: http://www.redalyc.org/articulo.oa?id=71710105

5. Scimago Institutions Rankings (SIR). SIR Iber Peru 2015 [Internet]. SIR c2015. Disponible en: http://www.scimagoir.com/pdf/iber_new/SIR Iber PER 2015 HE.pdf

6. Perú, Congreso de la República. Ley Universitaria. Ley № 30220. 09 de Julio del 2014.

7. Mejia MO, Veramendi-Espinoza L, Huerta-Collado YM, MontenegroIdrogo JJ. Baja publicación de investigaciones médico estudiantiles curriculares de una universidad peruana. Rev Peru Med Exp Salud Publica. 2014; 31(3):608. Disponible en: http://dx.doi.org/10.17843/ rpmesp.2014.313.107

8. Osada J, Loyola-Sosa S, Ruíz-Grosso P. Publicación de Trabajo de Conclusión de Curso de Estudiantes de Medicina de una Universidad Peruana. Revista Brasileira De Educação Médica. 2014; 38 (3): 308-313. Disponible en: http://dx.doi.org/10.1590/S0100-55022014000300004

9. Mayta-Tristán P, Cartagena-Klein R, Pereyra-Elias R, Portillo A, RodriguezMorales A. Apreciación de estudiantes de Medicina latinoamericanos sobre la capacitación universitaria en investigación científica. Rev Med Chile. 2013; 141: 716-722. Disponible en: http://dx.doi.org/10.4067/ S0034-98872013000600005

10. Urquia-Osorio $\mathrm{H}$, Henriquez-Marquez K, Vásquez Bonilla W, EstradaMendoza A, Rodríguez-Morales A. Producción científica de decanos de medicina y salud de universidades centroamericanas. Salud Pública de México. 2014; 56 (3): 243-244. Disponible en: http://www.scielo.org.mx/ scielo.php?script=sci_arttext\&pid=S0036-36342014000300008

11. Avila M, Rodríguez-Restrepo A. La importancia de la investigación en el pre-grado de medicina. Medwave. 2014; 14 (10): 1-7. Disponible en: https://doi.org/10.5867/medwave.2014.10.6032

12. Silva I, Espig H. La producción científica en estudiantes de medicina de una Universidad Autónoma en Venezuela". Comunidad y Salud. 2014; 12 (2): 39-50. Disponible en: http://ve.scielo.org/scielo.php?script=sci arttext\&pid=S1690-32932014000200007
13. Ortega-Loubon C, Zuñiga-Cisneros J, Castro F, Barría-Castro JM, Lalyre A, Silva $S$, y cols. Capacitación en investigación brindada a los estudiantes de medicina de la facultad de medicina de la Universidad de Panamá. 2013; 9 (3): 1-10. Disponible en: http://www.archivosdemedicina. $\mathrm{com} /$ medicina-de-familia/capacitacin-eninvestigacinbrindada-alosestudiantes-demedicina-dela-facultad-demedicina-de-launiversidaddepanam.php?aid $=625$

14. Purizaca-Rosillo N, Cardoza-Jiménez K, Herrera-Añazco P. Producción científica en una universidad pública peruana beneficiaria del canon. An Fac med. 2016; 77(1): 73-4. Disponible en: https://doi.org/10.15381/ anales.v77i1.11561

15. Atamari-Anahui N, Roque-Roque J, Robles-Mendoza R, Nina-Moreno $P$, Falcón-Huancahuiri B. Publicación de tesis de pregrado en una facultad de Medicina en Cusco, Perú. Rev Med Hered. 2015; 26: 217-221. Disponible en: https://doi.org/10.20453/rmh.v26i4.2707

16. Toro-Huamanchumo CJ, et al. Financiamiento de la investigación en pregrado en las facultades de medicina peruanas. Gac Sanit. 2016. Disponible en: http://dx.doi.org/10.1016/j.gaceta.2016.06.009

17. Garmendia F. Criterios para una reforma curricular en la Facultad de Medicina. CIMEL. 2002; 7 (1):10-2.

18. Molina-Ordóñez J, Huamaní C, Mayta-Tristán P. Apreciación Estudiantil Sobre La Capacitación Universitaria En Investigación: Estudio Preliminar. Rev Peru Med Exp Salud Pública. 2008; 25(3): 325-29. Disponible en: http://dx.doi.org/10.17843/rpmesp.2008.253.1283

19. Peralta-Heredia I, Espinosa-Alarcón P. ¿El dominio de la lectura crítica va de la mano con la proximidad a la investigación en salud? Rev Invest Clin. 2005; 57(6): 775-83. Disponible en: https://www.medigraphic.com/ cgi-bin/new/resumen.cgi?IDARTICULO=6508

20. Mayta-Tristán P. Enseñando a publicar desde el pregrado. Rev. Méd. Risaralda. 2013; 19 (1): 2-3. Disponible en: https://revistas.utp.edu.co/ index.php/revistamedica/article/view/8455/5207

21. Huamani C, Chavez-Solis P, Dominguez-Haro W, Solano-Aldana M. Producción científica estudiantil: análisis y expectativas. Rev Peru Med Exp Salud Publica. 2007, 24: 444-446. Disponible en: http://dx.doi. org/10.17843/rpmesp.2007.244.1146

22. Gutiérrez C, Mayta P. Publicación desde el Pre Grado en Latinoamérica: importancia, limitaciones y alternativas de solución. CIMEL. 2003; 8 (1):54-60. Disponible en: http://sisbib.unmsm.edu.pe/BVRevistas/cimel/ v08_n1/PDF/a10v8n1.pdf

23. Salinas J. Investigación en pregrado: dificultades y posibles soluciones. An. Fac. Med. 2008; 69 (3): 216. Disponible en: https://doi.org/10.15381/ anales.v69i3.1144

24. Arroyo-Hernández $\mathrm{CH}$, De la Cruz W, Miranda-Soberon UE. Dificultades para el desarrollo de investigaciones en pregrado en una universidad pública de provincia, Perú. Rev Peru Med Exp Salud Publica. 2008; 25(4):448-448. Disponible en: http://dx.doi.org/10.17843/ rpmesp.2008.254.1316 
25. Carvajal A. Importancia y reflexiones sobre la investigación y publicación científica desde pregrado. SCientifica. 2014; 12(1): 7-8. Disponible en: http://www.revistasbolivianas.org.bo/scielo. php?pid=s1813-00542014000100001\&script=sci_arttext

26. Carrillo-Larco R, Osada J. Promoción de la investigación: viviendo sin una cultura de publicación. Educ Méd. 2012; 15 (3): 131. Disponible en: http://doi.org/ 10.4321/S1575-18132012000300003

27. Frishman W. Student research projects and theses: should they be a requirement for medical school graduation? Heart Disease. 2001; 3: 140 144. Disponible en: https://www.ncbi.nlm.nih.gov/pubmed/11975783

28. Pamo O. Estado actual de las publicaciones periódicas científicas médicas del Perú. Rev Med Hered. 2005; 16(1): 65-73. Disponible en: https://doi.org/10.20453/rmh.v16i1.865

29. Neyra M, Berra M, Rodríguez A, Rodríguez R, Reyes $G$. La estrategia investigativa curricular en la carrera de medicina. Rev Cub Educ Med Sup. 1997; 11(2): 91 -100. Disponible en: http://scielo.sld.cu/scielo. php?script=sci_arttext\&pid=S0864-21411997000200003

30. Pachajoa H. Publicación de artículos originales desde el pregrado en una revista médica colombiana entre 1994-2004. CIMEL. 2006; 11(1):24-6. Disponible en: http://www.redalyc.org/articulo.oa?id=71711108

31. Sogi C, Perales A, Anderson A, Bravo E. El proceso de producción científica de los investigadores de la Facultad de Medicina, UNMSM. An Fac Med. 2002; 63(2):115-24. Disponible en: https://doi.org/10.15381/ anales.v63i2.1491

32. Mayta-Tristán P. ¿Quién es el autor? Aspectos a tener en cuenta en la publicación de artículos estudiantiles. CIMEL. 2006; 11(2):50-2. Disponible en: http://sisbib.unmsm.edu.pe/BVRevistas/cimel/v11_n2/ pdf/a02v11n2.pdf

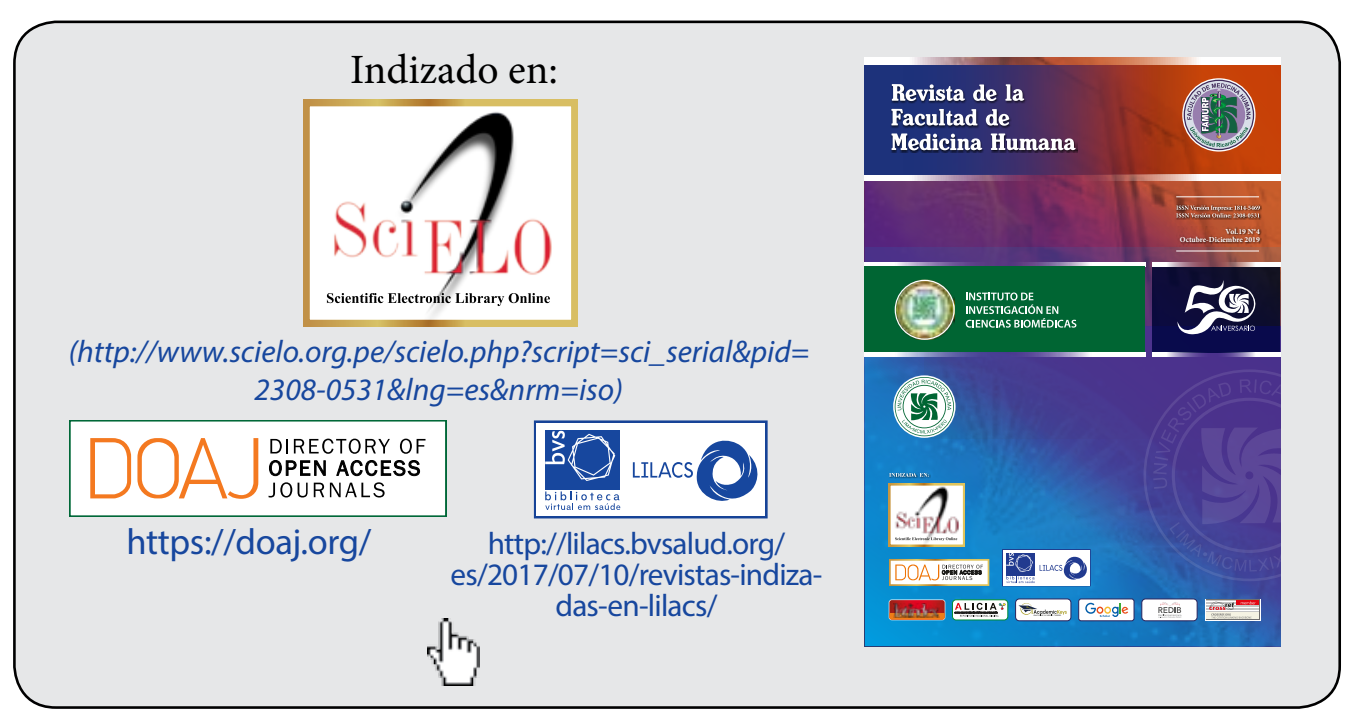

